Fournal of Medical Genetics (1972). 9, 356-380.

\section{8,XYYY: A New Syndrome?}

In 1965, Townes, Ziegler, and Lenhard reported a 5-year-old boy with a 48,XYYY chromosome complement. Mosaics with one cell line bearing more than $2 \mathrm{Y}$ chromosomes have been reported (Fraccaro, Taylor, and Bodian, 1962; Cox and Berry, 1967; van den Berghe, Verresen, and Cassiman, 1968), but to our knowledge the present case of $48, \mathrm{XYYY}$ is the second to be reported.

The introduction of fluorescent staining of chromosomes by Caspersson, Zech, and Johansson (1970) provided the means for specific identification of all 22 autosomes as well as the $\mathrm{X}$ and $\mathrm{Y}$. A recently described heterochromatin staining technique (Pardue and Gall, 1970) adds an additional means of identifying specific chromosomes using conventional light microscopy. The purpose of the present paper is to report a case that utilized these new techniques for the diagnosis.

\section{Case Report}

The propositus is a 9-year-old Caucasian boy born by Caesarean section to a 27-year-old, gravida 3 para 2, woman after an unremarkable 38-week gestation. The father was 27 years old at the time of the patient's birth. The parents have been in good health, and there is no family history of note apart from a cousin of the propositus (mother's sister's son) who at age 12 is no longer in school because of mental retardation. The cousin's diagnosis is unknown, and to our knowledge chromosome studies have not been performed. The patient's sisters (aged 10 and 12 years) and brother (aged 7 years) are in good health.

The boy weighed $3260 \mathrm{~g}$ at birth and had choking respirations with considerable mucus immediately postpartum. During the first weeks of life he was subject to episodes of choking. At 5 weeks he stopped breathing while being breast fed, and required patting on the back before he resumed breathing. Following this episode he was hospitalized with a diagnosis of upper-respiratory infection associated with choking spells and cyanosis. Chest $x$-ray showed atelectasis in the right base. At 4 months of age the patient was again hospitalized with a radiological diagnosis of bronchopneumonia. He had pneumonia again at the age of 4 years. A grade I/VI systolic murmur was re-

Received 10 November 1971. ported on an examination at 14 months of age. No murmur has been heard on subsequent examinations.

The development milestones have been significantly delayed. The propositus rolled over back to front at 6 months of age, sat without support at 8 months, crawled at 12 months, walked alone at 2 years, was toilet trained at $2 \frac{1}{2}$ years, and put 2 or 3 words together at about 3 years. The boy entered the first grade at age 7 and upon getting behind was referred for psychological testing. The school psychologist reported an IQ of 70 and stated that he had strong tendencies for impulsiveness and aggression.

The propositus was referred to us at age 9 years 2 months by his family doctor. Physical examination revealed a mildly retarded, alert, and cooperative boy $140 \mathrm{~cm}$ (75th centile) in height and weighing $35.4 \mathrm{~kg}$ (80th centile). The little fingers on both hands were markedly incurved due to wedge-shaped, hypoplastic middle phalanges and there was a modified single palmar crease bilaterally (Fig. 1). Dermatoglyphic findings included palmar proximal and distal axial triradii bilaterally with a total finger ridge count of 137 (Penroses

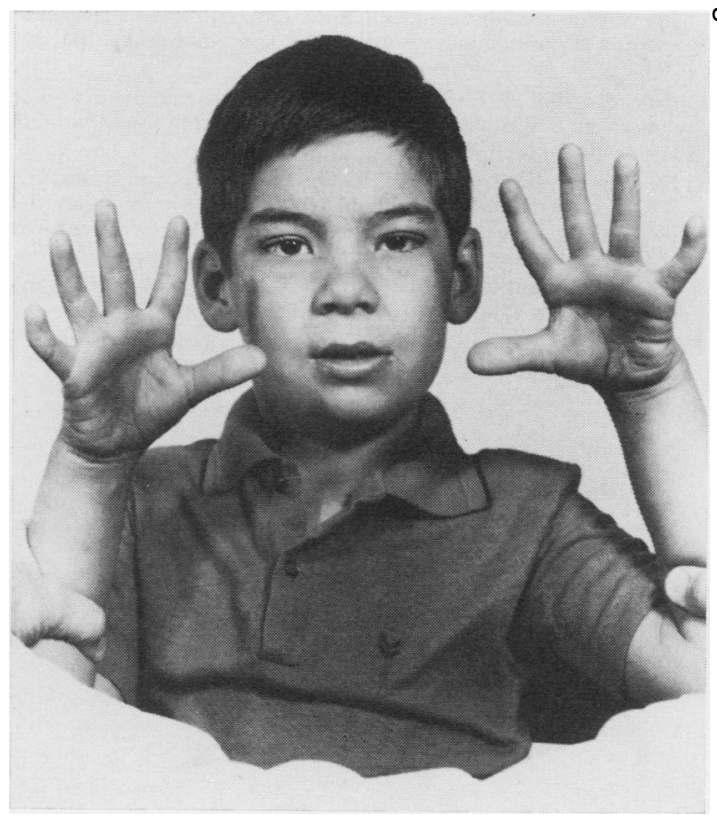

FIG. 1. The propositus with a modified simian crease on both hands and bilateral clinodactyly of the 5 th digits. The epicanthal folds and slight mongoloid slant of the eyes are found in family members and are not believed to be a part of the XYYY syndrome. 
1967). The genitalia were normal except for a small hydrocele on the left. The deep tendon reflexes were somewhat diminished bilaterally. The remainder of the physical and neurological examination was unremarkable.

An electroencephalogram and electrocardiogram were normal. Urinary steroid determination on a 24-hour specimen showed 17-ketosteroid and 17-ketogenic steroid values within normal limits for the propositus' age.

One of the presenting complaints from the parents was the stiff posture the patient assumed when running. An $x$-ray skeletal survey failed to reveal any bony abnormality to account for this observation. However, a hand and wrist bone age of between 4 and $4 \frac{1}{2}$ years was seen (Greulich and Pyle, 1959) as well as distal epiphyses of the 1st metacarpals and pseudoepiphyses at the proximal ends of the 2nd, 3rd, and 5th metacarpals bilaterally. The survey further revealed lack of calcification of patellar epiphyses, abnormal tarsal bone formation, and epiphyses at both ends of all metatarsal bones.

On psychological tests administered at our hospital the patient achieved a WISC verbal IQ of 70, performance IQ of 93, and full-scale IQ of 79. The patient is doing well in special classes and is not a behaviour problem. $\mathrm{He}$ is a loner and avoids fights, but does have occasional outbursts when placed under tension for too prolonged a period.

\section{Chromosome Studies on Peripheral Lymphocytes}

Chromosome spreads from peripheral lymphocytes were prepared using a modification of the micro-technique described by Moorhead et al (1960). Fifty metaphases were counted and 10 karyotypes were prepared. No variation from a count of 48 with 7 small acrocentrics was detected. Analysis of chromosome metaphases similarly prepared from the parents and sibs showed normal karyotypes.

\section{Chromosome Studies on Other Tissues}

A bone marrow aspirate was processed for chromosomes (Lam-Po-Tang, 1968). Fifty metaphases were counted, and 3 karyotypes were prepared. Again a count of 48 chromosomes with 7 small acrocentrics was found in all cells. Only one metaphase spread from skin fibroblasts was suitable for analysis. It contained 48 chromosomes with 7 small acrocentrics.

\section{Fluorescent Staining}

In that the identification of the extra small acrocentric chromosome was not clear from morphology or association, further studies were carried out using a modification (E. B. Lewis, personal communication) of the method of Pearson, Bobrow, and Vosa (1970) for fluorescent staining of interphase and metaphase chromosomes.
In the interphase studies, simple blood films on glass slides were stained with quinacrine dihydrochloride (Atebrin). The fluorescent interphase nuclei of peripheral leucocytes were examined under oil immersion for fluorescent bodies. We have found the term 'fluorosome' useful in describing the fluorescent body representing the distal ends of the long arms of the $\mathrm{Y}$ chromosome in interphase nuclei.
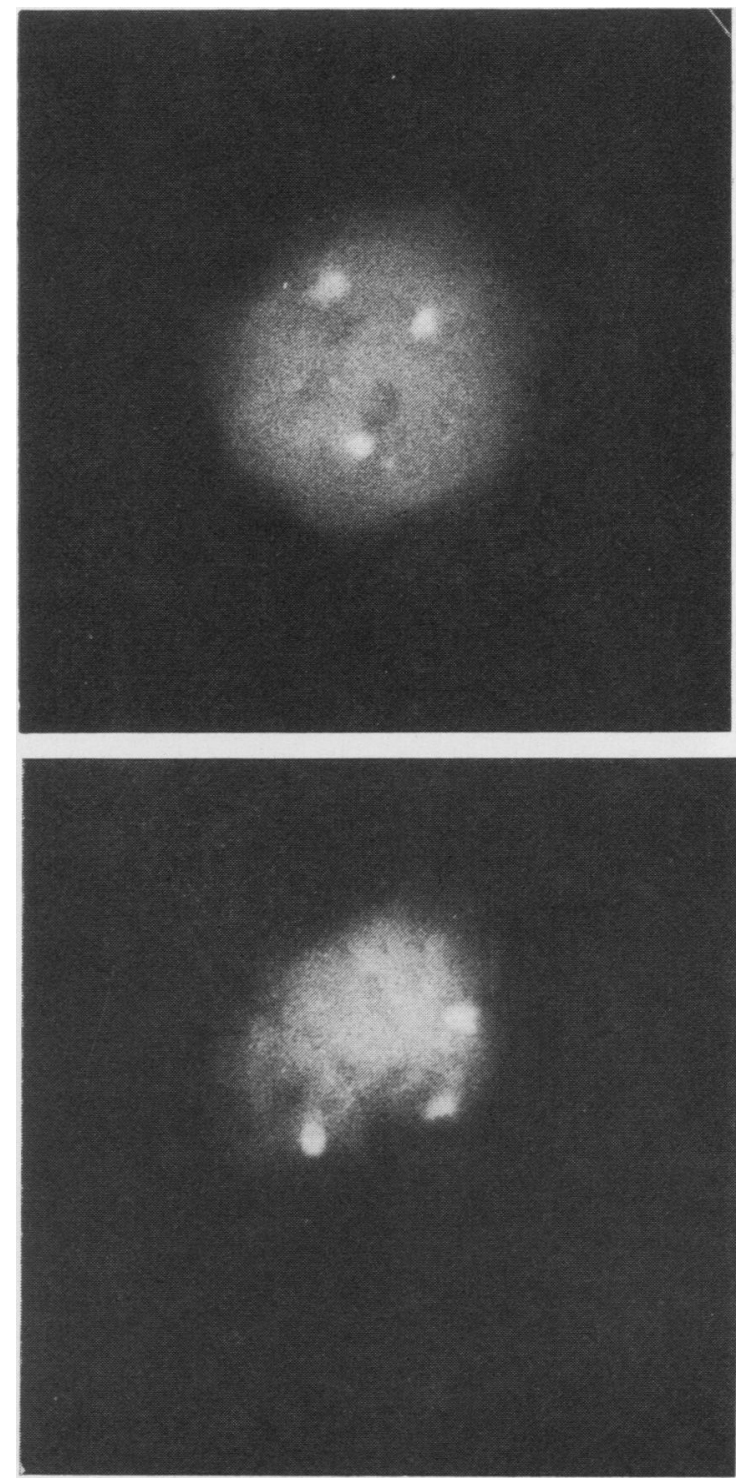

Fig. 2. Quinacrine-stained interphase lymphocyte and polymorphonuclear leucocyte nuclei each containing 3 'fluorosomes'. 
In the 354 interphase nuclei studied, $24(6.8 \%)$ contained 3 fluorosomes (Fig. 2), 109 (30.8\%) contained 2 fluorosomes, $141(39.8 \%)$ contained 1 fluorosome, and $80(22.6 \%)$ contained no fluorosomes. In XY male controls no cells were observed to have 3 fluorosomes and only rarely $(0.4 \%)$ were 2 fluorosomes seen-presumed artefacts or fluorescent segments on other chromosomes. Approximately $55 \%$ had 1 fluorosome.

An unstained chromosome preparation from peripheral leucocytes was also stained with quinacrine dihydrochloride. The distal ends of the long arms of 3 of the small acrocentrics fluoresced, identifying them as $\mathrm{Y}$ chromosomes (Fig. 3).

\section{Heterochromatin Staining}

Recently, Arrighi and Hsu (1971) reported a modification of the technique of Pardue and Gall (1970) for preferential Giemsa staining of heterochromatin in human chromosomes. Dr Hsu kindly stained a metaphase spread from a peripheral leucocyte of the propositus. The preparation shown in Figs. $4 \mathrm{a}$ and $\mathrm{b}$ distinguishes the $\mathrm{Y}$ chromosomes from the morphologically similar $\mathrm{G}$ group by staining the heterochromatin localized in the distal ends of the long arms of the $\mathrm{Y}$ chromosomes.

\section{Discussion}

A report is made of a 9-year-old boy who has 48 chromosomes. The phenotype did not suggest Down's syndrome and the 2 extra small acrocentric chromosomes were found to be $\mathrm{Y}$ chromosomes by fluorescent and heterochromatin stains.

In 1965, Townes et al reported that the first case of 48,XYYY had frequent upper-respiratory infections, mildly retarded psychomotor development with an IQ of 80, a left inguinal hernia, brownish teeth, valvular pulmonary stenosis, a normal neurological examination, and bilateral simian lines with otherwise unremarkable dermatoglyphic findings. In the present case, no heart lesion was detected, and the dentition appeared normal, but the other findings

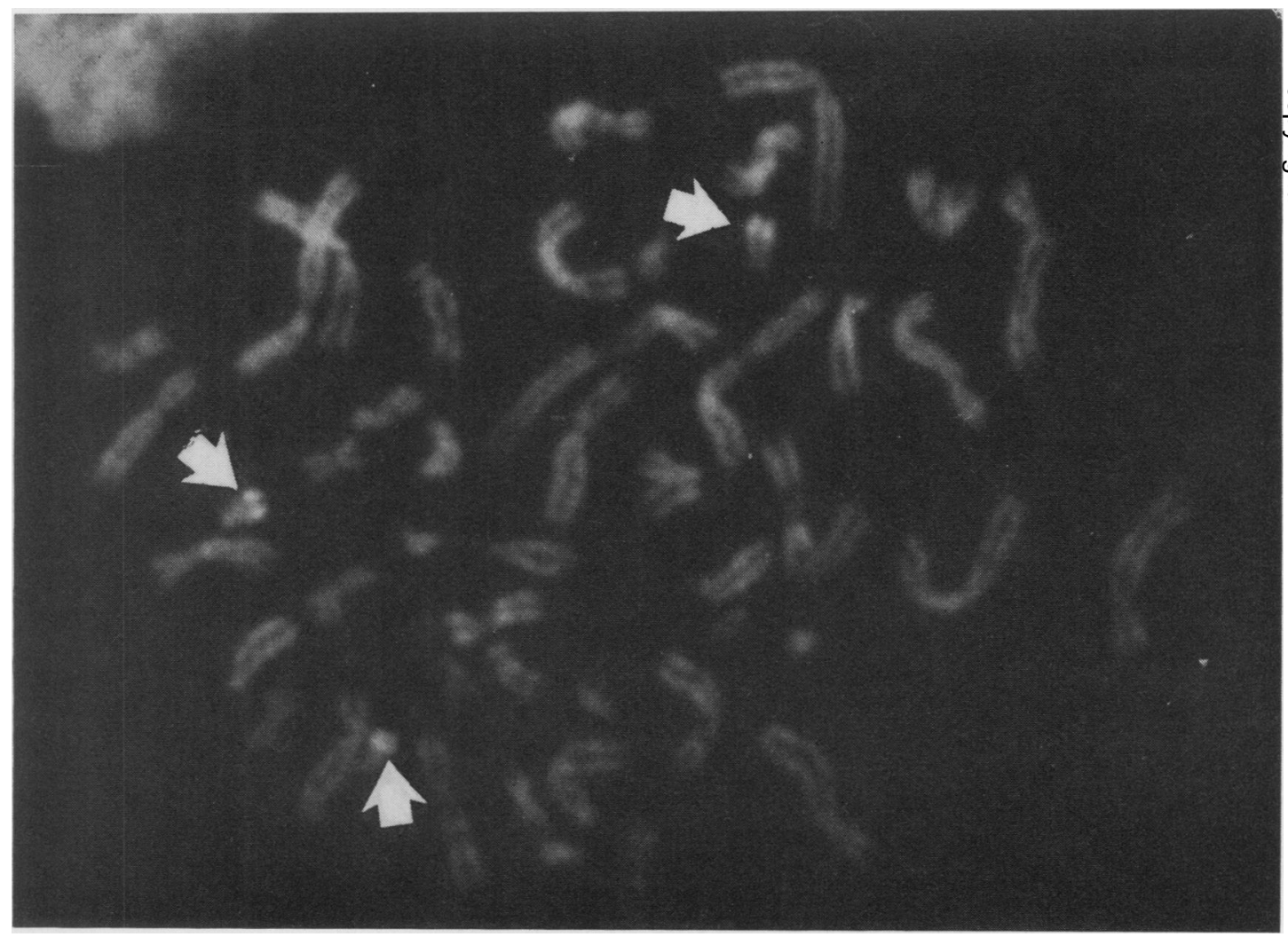

Fig. 3. A cluster of 48 quinacrine-stained metaphase chromosomes from a peripheral leucocyte showing the fluorescent distal ends of the $3 \mathrm{Y}$ chromosomes. 


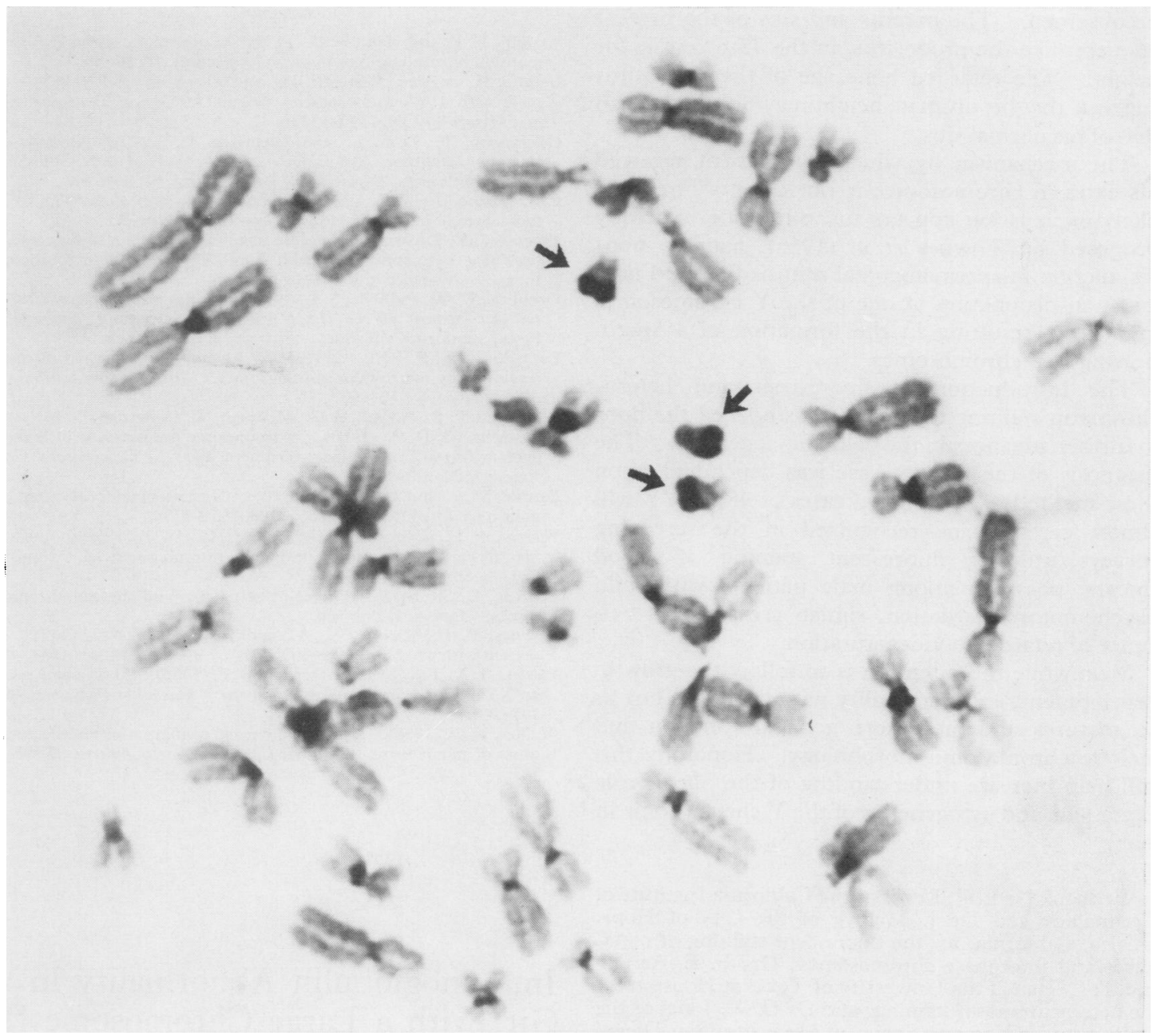

Fig. 4a

correlate fairly well. No mention of bone maturation was made in the first case reported.

By the propositus' chromosome morphology alone it was impossible to rule out a trisomy 21 in an XYY patient. However, the correlation for Down's syndrome computed from the dermatoglyphic findings according to the method of Walker (1958) was $-2 \cdot 67$. This together with a lack of the phenotypic changes noted in a reported case of trisomy 21 in an XYY patient (Uchida, Ray, and Duncan, 1966) made this diagnosis improbable even before the exact diagnosis was confirmed by fluorescent and heterochromatin staining techniques.

The ultimate size of XYYY individuals has yet to

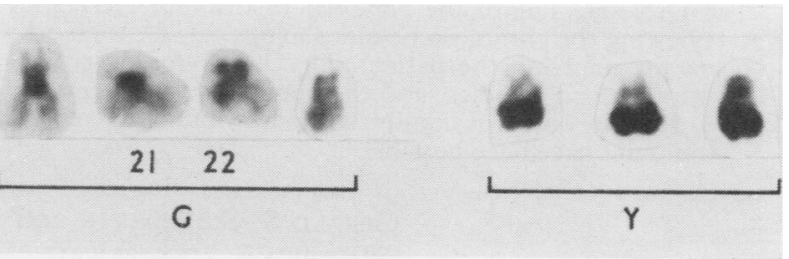

Fig. 4b

Frg. 4a. Heterochromatin stain of the 48 metaphase chromosomes from a peripheral leucocyte showing the preferential staining of the distal ends of 3 of the small acrocentric chromosomes, which identifies them as $\mathrm{Y}$ chromosomes.

Fig. 4b. Partial karyotype of the heterochromatin-stained chromosome metaphase of Fig. 4a. 
be observed. The parents and sibs of the present case are, like the propositus, in the 75 th centile for height. The retarded bone age of the propositus suggests that his ultimate height may be greater than that of his normal sibs.

The mechanism by which this patient received his extra $\mathrm{Y}$ chromosomes is not clear. The most likely explanation appears to be the one originally proposed by Townes et al (1965), namely, nondisjunction in spermatogonial mitosis followed by a 2nd non-disjunction of one of the $\mathrm{Y}$ chromosomes in meiosis resulting in the formation of a sperm bearing $3 \mathrm{Y}$ chromosomes.

The introduction of fluorescent and heterochromatin staining techniques has opened the door to further advances in the field of cytogenetics. The diagnosis of the present case was dependent upon these methods. Additional cases of 48,XYYY will almost certainly be recognized in the screening surveys utilizing fluorescent staining of blood smears, possibly among male patients with mild psychomotor retardation, simian creases, and evidence of retarded bone maturation.

Meanwhile the intention is to follow the growth, development, and personality pattern of this boy as he matures and later note gonadal function and meiotic chromosome morphology. Hopefully this will help increase understanding of the phenotypic expression and cytogenetics of the $\mathrm{Y}$ chromosome in man.

We thank Dr E. B. Lewis of the California Institute of Technology and Dr J. Melnyk of the City of Hope, Duarte, California, for the fluorescent staining of metaphase and interphase chromosomes; Drs F. E. Arrighi and T. C. Hsu of the University of Texas at Houston for the heterochromatin staining; and $\mathrm{Dr} O$. W. Jones of the University of California School of Medicine (San Diego) for culturing the skin fibroblasts. Thanks are also due to Loma Linda University physicians, Dr H. Schone for reviewing the dermatoglyphics, Dr I. Kuhn for the bone marrow aspiration, and Drs W. Stilson and H. Gorman for interpreting the $x$-rays. We acknowledge the help of Dr J. H. Tjio of the National Institute of Health in reviewing the chromosome morphology, Dr G. Richardson for referring the patient, Dr G. Joy for obtaining various blood samples, and the outstanding cooperation of this boy and his family without which this report would not have been possible.

GERALD S. SCHOEPFLIN and WILLARD R. CENTERWALL*

Department of Pediatrics, Loma Linda University School of Medicine, California 92354, USA

\footnotetext{
* Our laboratory is supported in part by grants from the Walter E. Macpherson Society and The National Foundation-March of Dimes.
}

REFERENCES

Arrighi, F. E. and Hsu, T. C. (1971). Localization of heterochromatin in human chromosomes. Cytogenetics, 10, 81-86.

Berghe, H. van den, Verresen, H., and Cassiman, J. J. (1968). A :-

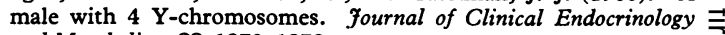
and Metabolism, 28, 1370-1372.

Caspersson, T., Zech, L., and Johansson, C. (1970). Analysis of human metaphase chromosome set by aid of DNA-binding fluorescent agents. Experimental Cell Research, 62, 490-492.

Cox, D. and Berry, C. L. (1967). A patient with $45 X O / 48 X Y Y Y$ mosaicism. Fournal of Medical Genetics, 4, 132-133.

Fraccaro, M., Taylor, A. I., and Bodian, M. (1962). A human intersex ('true hermaphrodite') with $\mathrm{XX} / \mathrm{XXY} / \mathrm{XXYYY}$ sex chromosomes. Cytogenetics, 1, 104-112.

Greulich, W. W. and Pyle, S. I. (1959). Radiographic Atlas of Skeletal Development of the Hand and Wrist. Stanford University Press, Stanford, California.

Lam-Po-Tang, P. R. L. C. (1968). An improved method of processing bone marrow for chromosomes. Scandinavian fournal of Haematology, 5, 158-160.

Moorhead, P. S., Nowell, P. C., Mellman, W. J., Battips, D. M., and Hungerford, D. A. (1960). Chromosome preparations of leukocytes cultured from human peripheral blood. Experimental Cell is Research, 20, 613-616.

Pardue, M. L. and Gall, J. G. (1970). Chromosomal localization of mouse satellite DNA. Science, 168, 1356-1358.

Pearson, P. L., Bobrow, M., and Vosa, C. G. (1970). Technique for identifying $\mathrm{Y}$ chromosomes in human interphase nuclei. Nature, 226, 78-80.

Penrose, L. S. (1967). Finger-print pattern and the sex chromosomes. Lancet, 1, 298-300.

Townes, P. L., Ziegler, N. A., and Lenhard, L. W. (1965). A patient with 48 chromosomes (XYYY). Lancet, 1, 1041-1043.

Uchida, I. A., Ray, M., and Duncan, B. P. (1966). 21 Trisomy with an XYY sex chromosome complement. Fournal of Pediatrics, 69, 295-298.

Walker, N. F. (1958). The use of dermal configurations in the diag nosis of mongolism. Pediatric Clinics of North America, 5, 531 543.

\section{Immunoglobulin Abnormality in a Girl with a Large Chromosome 18}

Feingold et al (1968) first reported a case of IgA deficiency associated with partial deletion of the long arm of chromosome 18. Since then IgA deficiency has been reported in several patients with different abnormalities of chromosome 18 namely, partial deletion of the long arm, short arm deletion, and a ring 18 chromosome. Long arm deletion of chromosome 18 has been recognized as a clinical syndrome (de Grouchy, 1969). Chromosome 18 presumably has a locus for the production of $\operatorname{IgA}$, and deletion of part of this chromosome results in deficiency in an immunoglobulin $A$. Hecht (1969), however, reported a case of 18 trisomy associated with a striking deficiency of serum $\operatorname{IgA}$, and some reports had no IgA deficiency (Borgaonkar

Received 10 February 1972. 